\title{
Method for detection of leads from Sentinel-1 SAR images
}

\author{
Dmitrii MURASHKIN ${ }^{1}$ Gunnar SPREEN, ${ }^{1}$ Marcus HUNTEMANN, ${ }^{1,2}$ \\ Wolfgang DIERKING ${ }^{2,3}$ \\ ${ }^{1}$ University of Bremen, Institute of Environmental Physics, Bremen, Germany \\ Email: murashkin@uni-bremen.de \\ ${ }^{2}$ Alfred Wegener Institute for Polar and Marine Research, Bremerhaven, Germany \\ ${ }^{3}$ Arctic University of Norway / CIRFA, Tromso, Norway
}

\begin{abstract}
The presence of leads with open water or thin ice is an important feature of the Arctic sea ice cover. Leads regulate the heat, gas and moisture fluxes between the ocean and atmosphere and are areas of high ice growth rates during periods of freezing conditions. Here, an algorithm providing an automatic lead detection based on synthetic aperture radar images is described that can be applied to a wide range of Sentinel-1 scenes. By using both the HH and the HV channels instead of single co-polarised observations the algorithm is able to classify more leads correctly. The lead classification algorithm is based on polarimetric features and textural features derived from the grey-level co-occurrence matrix. The Random Forest classifier is used to investigate the importance of the individual features for lead detection. The precision-recall curve representing the quality of the classification is used to define threshold for a binary lead/sea ice classification. The algorithm is able to produce a lead classification with more that $90 \%$ precision with $60 \%$ of all leads classified. The precision can be increased by the cost of the amount of leads detected. Results are evaluated based on comparisons with Sentinel-2 optical satellite data.
\end{abstract}

KEYWORDS: ice/atmosphere interactions, ice/ocean interactions, remote sensing, sea ice, sea-ice dynamics

\section{INTRODUCTION}

Sea ice covers a large part of the Arctic Ocean. It works as insulation between relatively warm ocean water and cold air in winter. One of the important features of the ice cover is the presence of sea-ice leads. Leads are areas with open water or thin ice, which are usually of elongated shape. They appear as result of ice fracturing due to shear and divergence stresses in the sea-ice cover. These stresses are forced by the ocean currents, tides and to a large degree by winds in the atmosphere. Leads regulate the heat, gas and moisture exchange fluxes between the ocean and the atmosphere and are places of increased sea-ice production, during periods of freezing conditions. Hence, the spatial and temporal distributions of leads are of interest for climate studies (Maykut, 1978; Wang and others, 2016). Furthermore, the mapping of sea-ice leads plays an important role for navigation providing an easier way for vessels through the pack ice. Also, leads are areas of increased biological activity in the ocean like phytoplankton blooms (Assmy and others, 2017). The life of Arctic animals (e.g. walruses, polar bears, birds) is often tied to leads.

Due to harsh weather conditions in the Arctic, capabilities for field studies of sea ice are limited. Remote-sensing methods can provide measurements in the Arctic covering large areas. Airborne and satellite instruments allow one to receive data with different spatial and temporal resolutions. Several methods were developed for discrimination of sea ice and water based on remote-sensing observations taken in the visible, infrared, and microwave parts of the electromagnetic spectrum. They are described in the following four paragraphs.

A large number of advanced very-high resolution radiometer (AVHRR) infrared images were analysed by Lindsay and
Rothrock (1995) to determine lead characteristics. The lead detection algorithm is based on the concept of 'potential open water', a scaled version of the surface temperature or albedo that weights thin ice by its thermal or brightness impact. A threshold was set to discriminate open water from sea ice. The resolution of the data is about $2-3 \mathrm{~km}$. In the studies conducted by Willmes and Heinemann (2015, 2016), MODIS thermal infrared imagery was used for lead detection. The method is based on a threshold applied to MODIS images with the background subtracted. The resulting maps with resolution of $1 \mathrm{~km}$ contain three classes: sea ice, leads and lead-like structures. Both the AVHRR and MODIS-based methods are affected by cloud contamination.

The cloud influence is significantly reduced for observations in the microwave spectrum, where weather conditions have little influence on measurements. Lead detection based on microwave altimetry was studied by Wernecke and Kaleschke (2015) using data from CryoSat-2. The algorithm to discriminate leads from ice is based on the maximum power, the pulse peakiness, and other parameters (e.g. the leading edge width, the trailing edge width, the stack standard deviation, and the stack excess kurtosis) of the reflected altimeter signal.

Estimations of lead concentrations and their orientation statistics were shown by Röhrs and Kaleschke (2012) and Bröhan and Kaleschke (2014) based on observations of the passive microwave imager AMSR-E. For AMSR-E data on a $6.25 \mathrm{~km}$ grid, they detect leads wider than $3 \mathrm{~km}$, which results in a detection of $50 \%$ of the lead area that can be seen on MODIS optical images.

Synthetic aperture radar (SAR) is able provide high-resolution data with large spatial and temporal coverage. It is widely used for sea-ice-type classification and ice-water 
discrimination (Dierking, 2010, 2013). Ivanova and others (2016) used a threshold approach on the $\mathrm{HH}$-band $(\mathrm{HH}$ : transmitting and receiving in horizontal polarization) of the ENVISAT ASAR instrument for water-ice discrimination. To improve the quality of object classification the directly measured data (e.g. backscatter intensities) are often extended with textural features. Ice-water classification based on dual-polarised SAR images (RADARSAT-2) with the additional texture information is described by Leigh and others (2014). The use of a support vector machine on the features based on the grey-level co-occurrence matrix (GLCM) (Haralick and others, 1973) is suggested. This combination was previously used for sea-ice-type classification by Liu and others (2015), Korosov and Park (2016). A similar approach is used by Zakhvatkina and others (2017) for icewater discrimination. A neural network with texture features based on the GLCM was used for classification of ENVISAT SAR images by Zakhvatkina and others (2013). Another method that provides complementary information to the backscatter intensity is based on polarimetric features (e.g. Moen and others, 2015). Polarimetric features are used for sea-ice classification (e.g. Ressel and others, 2016), iceberg detection (Dierking and Wesche, 2014) and oil spill recognition (e.g. Brekke and others, 2014).

In this study, Sentinel-1 dual-channel C-band SAR measurements (co- and cross-polarised modes, $\mathrm{HH}$ and $\mathrm{HV}$ ) are used. It should be noticed that several definition of a lead can be found in the literature (Weeks, 2010). Although leads often defined as elongated areas with open water, here we do not consider the shape of detected objects. The automatic lead detection algorithm we have developed is based on the analysis of backscatter amplitude and texture and, therefore, thin ice and open water between ice flows might be classified as leads. We combine polarimetric and texture features to produce sea-ice lead maps that, if combined, can cover the complete European Arctic. We investigate the optimal number of texture features for lead classification and provide the precision-recall curve as a classification quality metric. Results are evaluated by highresolution $(10 \mathrm{~m})$ optical data from Sentinel-2.

\section{DATA AND METHODS}

\section{Satellite data}

The data used in the present study are listed in Table 1. For this study, we focused on two satellite constellations: Sentinel-1 SAR data are used for the lead detection and
Sentinel-2 optical data as evaluation dataset. The data are available at the Copernicus Open Access Hub (scihub.copernicus.eu). The Copernicus Sentinel-1 mission currently comprisestwo satellites with a SAR as primary instrument. In the Extra Wide swath mode, SAR images are acquired at both $\mathrm{HH}$ and $\mathrm{HV}$ polarisations. The backscatter of the electromagnetic wave transmitted at $5.4 \mathrm{GHz}$ frequency at horizontal polarisation is received and decomposed into horizontal $(\mathrm{HH})$ and vertical $(\mathrm{HV})$ polarisation components. The swaths width is $400 \mathrm{~km}$, the resolution is $93 \times 87 \mathrm{~m}$ (pixel size is $40 \times 40 \mathrm{~m}$ ). The typical size of a Sentinel- 1 image is $10000 \times 10000$ pixels. Preprocessing of the data includes thermal noise removal, incidence angle correction and speckle noise filtering. The flowchart of the lead detection algorithm is shown in Figure 1. Thermal noise and correction parameters, which are provided in the auxiliary data, are applied to the SAR images according to the equation given in the Sentinel-1 processing chain documentation (ESA, 2016):

$$
\sigma=\frac{\left(\text { pixel value }{ }^{2}-\text { noise }\right)}{\gamma^{2}}
$$

where pixel value is the amplitude of backscatter of the original $\mathrm{HH}$ or $\mathrm{HV}$ band, noise is the intensity of the thermal noise and $\gamma$ is a calibration coefficient, noise and $\gamma$ are provided in the metadata. In the next step, the corrected backscatter is translated to $\mathrm{dB}$ by application of $\log _{10}$. To prevent infinitely low values a threshold of $1 / \max (\gamma)$ has been applied as a limit. In this way, the thermal noise is removed from the SAR data, but the so-called scalloping noise remains. The effect of the scalloping noise is mainly visible over the open ocean and therefore can be masked out with a sea-ice mask. A sea-ice mask can be retrieved by applying a threshold to a sea-ice concentration product. In this study, we use a $20 \%$ sea-ice concentration threshold for the ASI AMSR-2 algorithm [seaice.uni-bremen.de] (Spreen and others, 2008).

The last column gives the fraction of the satellite scene in per cent that is used for the classification training and testing.

Backscatter of sea ice in the $\mathrm{HH}$ band of SAR data is known to depend on the elevation angle (and, consequently, incidence angle), which therefore should be taken into account. A linear regression of $\mathrm{HH}$ backscatter versus elevation angle is used. The regression coefficients are derived from a set of 16 extra wide swath mode products with homogeneous areas acquired over sea ice. These products cover the entire range of incidence angles used in the extra wide

Table 1. List of Sentinel- 1 and -2 products used in the study

\begin{tabular}{|c|c|c|c|c|}
\hline Satellite & Sensor & Acquisition & Product type & Use in the study \\
\hline Sentinel-1 & SAR & 1 Oct $2015 ; 07: 36$ & S1A_EW_GRDM_1SDH & $25 \%$ training, $75 \%$ test \\
\hline Sentinel-1 & SAR & 28 Oct $2015 ; 14: 32$ & S1A_EW_GRDM_1SDH & $25 \%$ training, $75 \%$ test \\
\hline Sentinel-1 & SAR & 31 Oct $2015 ; 16: 35$ & S1A_EW_GRDM_1SDH & $25 \%$ training, $75 \%$ test \\
\hline Sentinel-1 & SAR & 4 Jan 2016; 02:01 & S1A_EW_GRDM_1SDH & $25 \%$ training, $75 \%$ test \\
\hline Sentinel-1 & SAR & 1 Feb $2016 ; 11: 15$ & S1A_EW_GRDM_1SDH & $25 \%$ training, $75 \%$ test \\
\hline Sentinel-1 & SAR & 3 Feb 2016; 22:30 & S1A_EW_GRDM_1SDH & Evaluation \\
\hline Sentinel-1 & SAR & 2 Aug 2016; 13:18 & S1A_EW_GRDM_1SDH & Evaluation \\
\hline Sentinel-2 & optical & 2 Aug 2016; 14:17 & S2A_OPER_PRD_MSIL1C_PDMC & Evaluation \\
\hline Sentinel-1 & SAR & 10 Apr 2017; 04:28 & S1A_EW_GRDM_1SDH & Evaluation \\
\hline Sentinel-2 & optical & 10 Apr 2017; 12:06 & S2A_MSIL1C & Evaluation \\
\hline
\end{tabular}




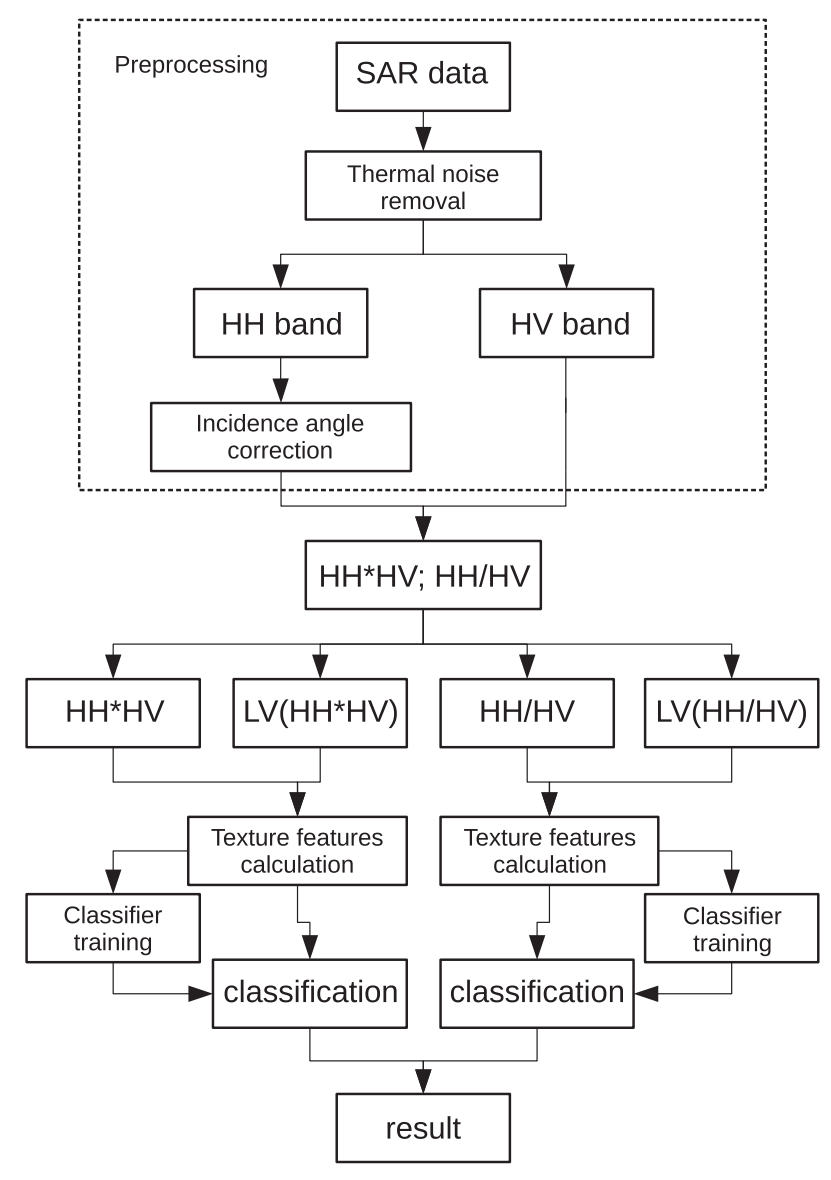

Fig. 1. Processing flowchart; LV stands for local variability (image with background subtracted).

swath mode. The resulting equation for the incidence angle correction is

$$
\sigma_{\text {new }}=\sigma+0.049 \cdot(\theta-\min (\theta))
$$

where $\sigma_{\text {new }}$ is the backscatter after the correction, given in $\mathrm{dB}$, and $\theta$ is elevation angle in degrees. The HV band does not reveal a significant sensitivity to the incidence angle; therefore, the correction is done only for the $\mathrm{HH}$ band. The next step of the preprocessing is the reduction of speckle noise. A bilateral filter is applied, which is an edge-preserving filter known to have good performance in reducing speckle noise (Tomasi and Manduchi, 1998; Alonso-Gonzalez and others, 2013). The size of the square window of the filter was set to 5 pixels. With this the preprocessing of the Sentinel- 1 data is finished.

The second source of satellite data used in this study are observations from the Sentinel-2 satellite constellation carrying a multispectral instrument with 13 bands in the visual and near-infrared spectral range. Images with a spatial resolution of $10 \mathrm{~m}$ for visual and $20 \mathrm{~m}$ for near-infrared are used for comparison with SAR images to evaluate the results of the lead detection algorithm.

\section{Training and evaluation datasets}

To train the classifier datasets are needed with correctly identified objects, i.e. in our case leads with thin ice or open water. We use a manual classification of six Sentinel-1 scenes that cover two different typical lead appearance types: dark and bright leads (three scenes for each of the two cases). These scenes are taken from different times of the year (Table 1). No scene from summer was used for training datasets because during the melt season melt ponds can have similar signature in SAR backscatter as leads with open water. To evaluate the results of the classification on independent data, two cases of overlapping Sentinel-1 SAR and Sentinel-2 optical data are used (Table 1). Although images in one of the cases were taken in August, there is no evidence of melt ponds presence on them. Hence, the melt season is excluded from the study.

Leads with open water or thin ice in most cases have low surface roughness and therefore have low backscatter values on both SAR bands, $\mathrm{HH}$ and HV. They appear dark on the optical image used for evaluation as well. This case is represented in the first of the two evaluation datasets. Figs $2 a, b$ and c show the $\mathrm{HH}$, the $\mathrm{HV}$ and the product of SAR bands $\mathrm{HH} \cdot \mathrm{HV}$, respectively, and Figure $2 \mathrm{~d}$ the Sentinel-2 image of the corresponding area taken in the visible spectrum. The time difference between the acquisitions is $7 \mathrm{~h} 40 \mathrm{~min}$. Most areas covered with leads appear dark on all images. Only around edges of leads thin crushed ice with a high surface roughness can appear bright while on the image taken in visible spectrum the same thin crushed ice is transparent. The assumption for using the band product $\mathrm{HH} \cdot \mathrm{HV}$ is that if either the $\mathrm{HH}$ or HV band show low backscatter intensities the band product will have low values.

Leads with open water, however, can appear bright on $\mathrm{HH}$ band under high incidence angles if wind roughens the water surface (Scharien and Yackel, 2005). In the HV band, leads appear dark under the same conditions. Thin sea ice with frost flowers in leads can have high backscatter values in both HH and HV bands (Nghiem and others, 1997; Dierking, 2010; Isleifson and others, 2014) and therefore can look like pressure ridges in C-band images. They might not always be classified correctly here. However, lead detection from the HV band is more prone to errors since the backscatter intensity at HV band is low and often close to the noise floor. An example of the situation when leads have high $\mathrm{HH}$ band backscatter intensities is shown in Figs 3a, b and $\mathrm{d}$ for Sentinel-1 HH, HV and Sentinel-2, respectively. Leads that are clearly observed in the optical data look brighter than the surrounding sea ice at HH band from SAR and darker in the HV band. In the band ratio $\mathrm{HH} / \mathrm{HV}$ image leads appear bright if $\mathrm{HH}$ is high and $\mathrm{HV}$ is low.

To account for these two different situations, we therefore split the lead classification algorithm into two parts: the first one detects leads that appear dark on both SAR bands, the second one is used for cases with high backscatter values at $\mathrm{HH}$ band (compare also the two branches in the flowchart in Fig. 1). In the last step, both outputs are merged to produce the final lead map.

Two cases of overlapping Sentinel- 1 and -2 data, presented in Figures 2 and 3, are used for the evaluation of our algorithm. Here leads are marked by hand on the SAR dataset taking into account the corresponding optical Sentinel-2 images to confirm the validity of the selected leads.

For the training of the algorithm six other independent Sentinel-1 scenes are used (Table 1). They also represent the two cases of dark and bright leads in the $\mathrm{HH}$ band, respectively. Three scenes are used to train the dark lead classifier and three other scenes to train the bright lead classifier. No optical Sentinel-2 data are available for these six scenes. Therefore, leads were identified and marked 

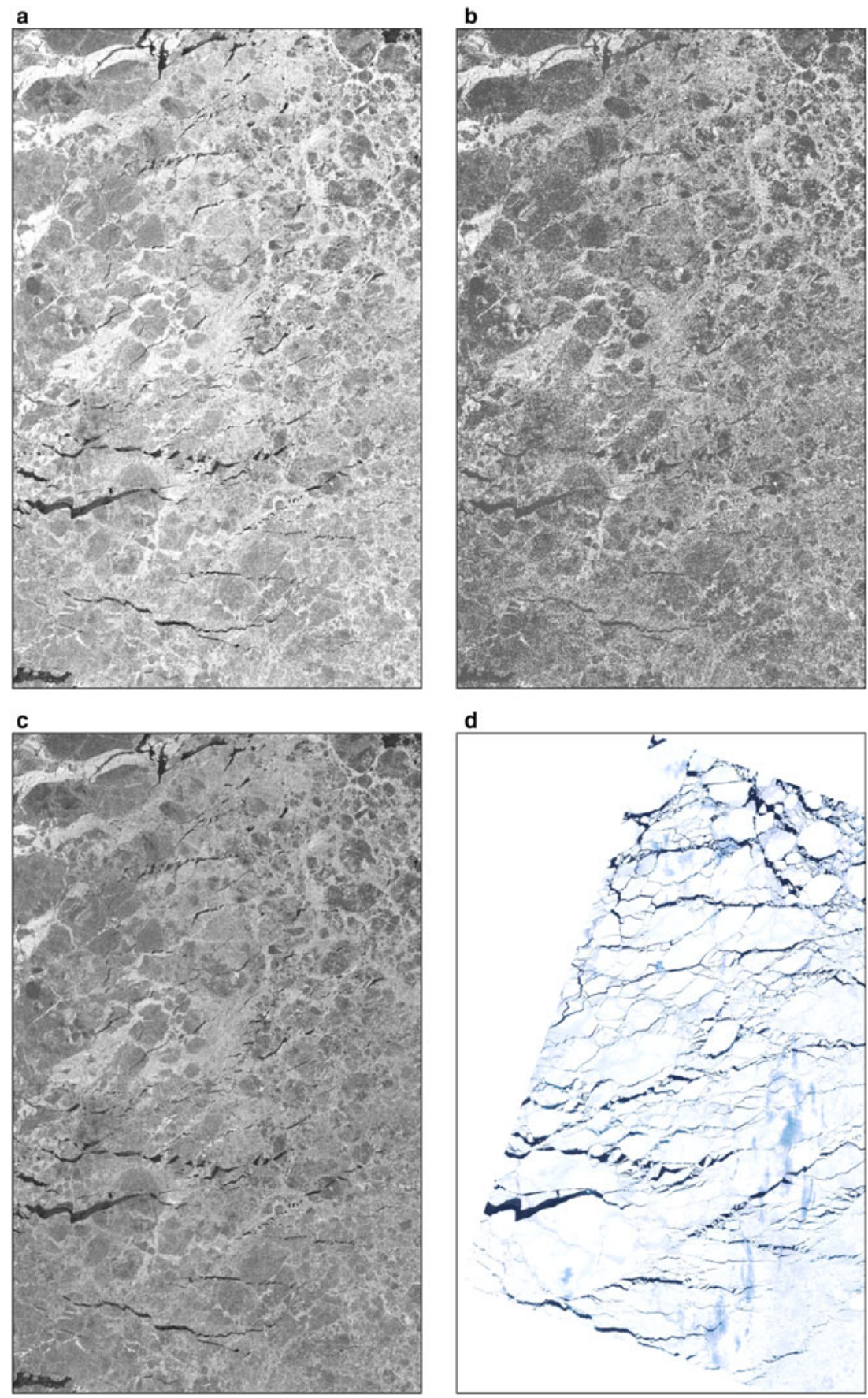

Fig. 2. Panels (a) and (b) are HH and HV bands. (c) Product of the HH and HV bands (HH·HV) of the SAR scene taken on 10 April 2017 , west of the Franz Josef Land. (d) Optical data from Sentinel-2 taken on 10 April 2017, west of the Franz Josef Land.

manually in the six Sentinel-1 scenes without the additional support from optical data. The later six images are used as training and test datasets, final evaluation is performed on SAR images which overlaps with optical data shown in Figures 2 and 3.

\section{Texture and polarimetric features}

In addition to the measured $\mathrm{HH}$ and HV backscatter intensities, the feature space for object classification in SAR images can be extended by two methods: polarimetric and texture features. Polarimetric features can be used for SAR products containing at least two out of the four Stokes vector components whereas texture features can be calculated for a single polarisation band. Here we combine the two approaches for Sentinel-1 dual-polarisation data. Products of the Sentinel-1 extra wide swath mode used in the Arctic contain co- and cross-polarised bands $(\mathrm{HH}$ and $\mathrm{HV}$ ). As only the amplitude but not phase is available for our data source, the number of available polarimetric features is restricted. In this study, the product $\mathrm{HH} \cdot \mathrm{HV}$ and the ratio $\mathrm{HH} / \mathrm{HV}$ are used as polarimetric features. The co-polarisation ratio $\mathrm{HH} / \mathrm{VV}$ and real part of the co-polarisation crossproduct is widely used for the SAR image classification Brekke and others (2016). It have shown good performance in discriminating open water from sea ice (Ressel and others, 2016). In the absence of VV, we use HV instead. The cross-polarisation ratio was used for classification of SAR images by (Karvonen, 2014) Bright, wind roughened leads in $\mathrm{HH}$ appear dark in HV (Fig. 3), which will cause 

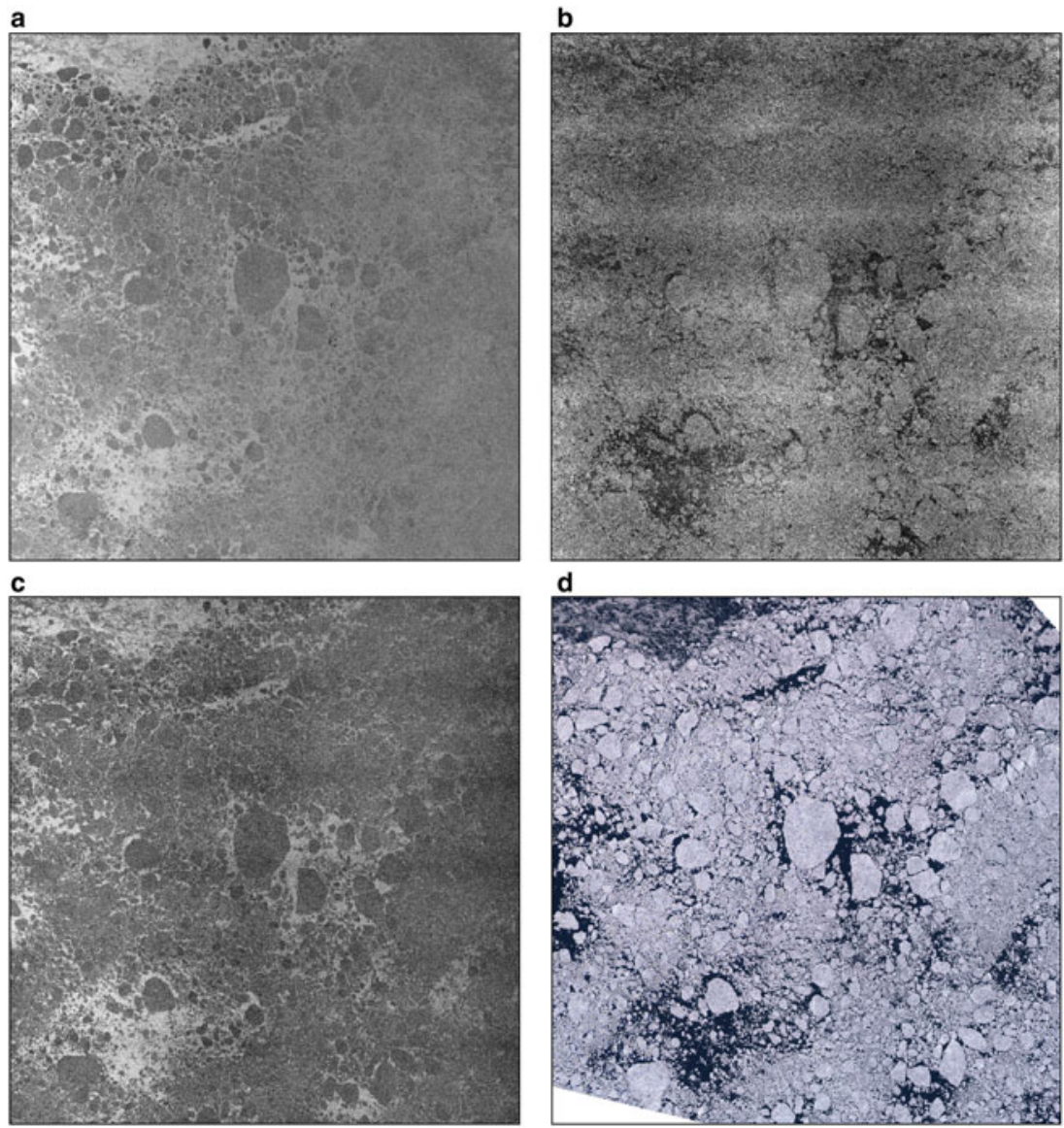

Fig. 3. Panels (a-c) are HH, HV bands and band ratio, respectively, of a Sentinel-1 SAR scene taken on 2 August 2016, between Svalbard and Franz Josef Land; (d) Optical data from Sentinel-2 taken on 2 August 2016, between Svalbard and Franz Josef Land.

high values in $\mathrm{HH} / \mathrm{HV}$ and this is the case we like to detect with the band ratio. The classification for dark leads is based on the $\mathrm{HH} \cdot \mathrm{HV}$ product, which will be low if one of the channels is low, i.e. dark. However, because of the low signal-to-noise ratio in the HV band the classification based on the band product is also compared with the classification based on the $\mathrm{HH}$ band alone to quantify if there is a benefit from the use of both bands.

Therefore, the $\mathrm{HH}$ band, the $\mathrm{HH} \cdot \mathrm{HV}$ product and the $\mathrm{HH} /$ $\mathrm{HV}$ ratio are used as input for the following texture analysis. Figures 2c and 3c show examples of the band product and ratio, respectively.

Texture features based on the GLCM are widely used for classification of SAR data (Haralick and others, 1973; Leigh and others, 2014; Liu and others, 2015; Zakhvatkina and others, 2017). The complexity of texture feature calculation depends on the size of the chosen sliding window and the number of grey levels of the input image. Here a discretisation into 16 grey levels has been chosen as trade-off between conservation of details and computational cost. GLCMs are then calculated for a sliding window of $9 \times-$ pixel size with 1 pixel step size. A bilinear weighting within the sliding window was applied, so that pixels which are closer to the middle of the window have higher weight for the GLCM computation.

The 12 GLCM features we use in this study are listed in Table 2. Definitions of the features are given by Haralick and others (1973). Some GLCM features depend on the image brightness (in our case the value of the $\mathrm{HH}$ and $\mathrm{HV}$ product or ratio). This means that the absolute value of the pixel brightness influences these texture features. Since leads are often darker than the surrounding sea ice, the difference between the original image and a low-pass filtered version of the image is calculated to show the small-scale backscatter variations. A bilateral filter with a $25 \times$-pixel sliding window is applied to the preprocessed original image and subtracted from the non-filtered image. In this way, the local backscatter variability is emphasised and is used further as an additional information for the classification. Afterwards GLCM texture features are calculated both

Table 2. Twelve GLCM features are used in the study

\begin{tabular}{ll}
\hline$N$ & Texture feature \\
\hline 1 & Angular second moment (ASM), \\
2 & Entropy, \\
3 & Contrast, \\
4 & Sum of squares: variance, \\
5 & Inverse difference moment (homogeneity), \\
6 & Correlation, \\
7 & Sum average, \\
8 & Sum variance, \\
9 & Sum entropy, \\
10 & Difference variance, \\
11 & Difference entropy, \\
12 & Information measure of correlation.
\end{tabular}

Definitions of the texture features are given by Haralick and others (1973). 
for (i) the original image and (ii) for the small-scale variations of the image. In this way, two sets of texture features are produced for each of two input polarimetric features (i) band product and (ii) band ratio, and for the $\mathrm{HH}$ band, and further analysed (see also flowchart in Fig. 1).

\section{Supervised learning algorithms}

We use the Random Forest Classifier (Breiman, 2001) implemented in scikit-learn library [http://scikit-learn.org/stable/ index.html] (Pedregosa and others, 2011) for sea-ice lead detection. It is an ensemble method that constructs a set of decision trees. Each of these trees is trained on a different subset of data points and features. The decision made by each tree is weighted to provide the final result. The method has been proven to have good quality of classification and at the same time high computational speed. One of the advantages of the classifier is its internal metric for feature importance, which gives information on the frequency of use of each of the input features. Another advantage of the classifier is its capability to perform not only binary, but also probabilistic classification. The probability of pixels to belong to a class can afterwards be translated to binary classification based on a threshold. The default behaviour is the use of a threshold of $50 \%$ probability. Different thresholds can be applied to adjust the result of classification. While there are several metrics to evaluate the quality of an algorithm, the most widely used metric is accuracy (Eqn (3) below). Although alone it might be unrepresentative for the case when the size of one class is considerably larger than the size of the other class. Leads usually occupy a few per cent of sea-ice area in the Arctic (Steffen, 1991), so that additional metrics should be used for quantifying the classification performance.

Precision and recall scores are defined by Fawcett (2006) and are used in the present study altogether with accuracy.

$$
\begin{gathered}
\text { accuracy }=\frac{T P+T N}{T P+F P+T N+F N} \\
\text { precision }=\frac{T P}{T P+F P} \\
\text { recall }=\frac{T P}{T P+F N}
\end{gathered}
$$

where $T P$ stands for true positive (pixels correctly classified as a lead), $T N$ - true negative (pixels correctly classified as not being a lead), FP - false positive (pixels that are not leads but are classified as a lead), $F N$ - false negative (pixels that are leads but are classified as 'not lead') predictions. The sum $T P+F P+T N+F N$ equals to the total number of pixel in the image. Accuracy can be explained as amount of correctly classified pixels over the total number of pixels. Precision is the amount of correctly classified pixels of the given class over the total number of pixels classified as the given class. Hence, $(1-$ precision $)=\frac{F P}{T P+F P}$ is the number of sea-ice pixels misclassified as a lead over the total amount of pixels classified as leads. The recall rate characterises how complete the classification is, that is the number of samples classified correctly over the total number of samples of this class. These three scores provide the needed information on the quality of a given class classification. They can aid to make decisions on how many features are needed for the classifiers.
To describe what probability threshold gives the best results for a probabilistic classifier, the receiver operating characteristic curve and the precision-recall curve are widely used (Fawcett, 2006; Davis and Goadrich, 2006). The receiver operating characteristic, for example, was applied for the lead detection algorithm described by Wernecke and Kaleschke (2015). Here we use the precision-recall curve to decide for an optimised binary threshold value for the Random Forest Classifier. This will be presented in the results section.

Two main parameters of the classifier that influence classification quality and computing time are the number of trees and the maximal depth of each tree. To choose the most suitable values for these parameters the six training SAR scenes, where leads were marked without support from optical data, have been used. The evaluation with the additional optical scenes will be presented later.

The two SAR datasets for the 'dark' and the 'bright' lead classifiers (three SAR scenes each) with manually identified leads have been randomly split into a training (25\% of the data) and a test dataset ( $75 \%$ of the data) each. Two classifiers are trained on the scenes where leads appear dark in the $\mathrm{HH}$ band: one is based on the $\mathrm{HH}$ band, another one is based on the $\mathrm{HH} \cdot \mathrm{HV}$ product. Results of the two classifiers are compared later. The third classifier is trained using the $\mathrm{HH} / \mathrm{HV}$ band ratio based on the scene where leads appear bright in the $\mathrm{HH}$ band. Each of the three classifiers are trained on the corresponding training dataset and then they are evaluated on the training and test datasets. A number of trees equal to 64 has been chosen for balance between efficiency and computing time. The maximal depth of trees was set to 15 to prevent overfitting and decrease computing time.

To decide how many texture features give positive benefit for the classification quality and to remove features that are not needed a so-called recursive feature elimination (RFE) is carried out. 12 texture features for the band data, 12 texture features for the small-scale variations of the band, and the original (preprocessed) band data altogether constitute 25 features which are used in the RFE. The Random Forest Classifier provides the rank of importance for all features used in the lead classification. Recursively, now after each training and classification the number of features is reduced by one and the training and the classification started again. After every step the texture feature with the lowest importance according to the classifier's metric (i.e. the feature least used) is eliminated. Accuracy, precision and recall are calculated to estimate the classification quality of the algorithm on the given subset of features. The operation is repeated until one feature is left. To calculate the three quality metrics a binary classification based on $50 \%$ probability threshold is used. Based on this experiment the optimal number of features can be chosen, which is presented in the next section.

\section{RESULTS}

\section{Optimal number of texture features for classification}

The procedure for texture feature ranking described in the last section was carried out three times: one time for the band product data $(\mathrm{HH} \cdot \mathrm{HV})$, the second one for the $\mathrm{HH}$ band data and the third one for the band ratio $(\mathrm{HH} / \mathrm{HV})$. The band product and the $\mathrm{HH}$ band are used for the training dataset where leads appear dark and the band ratio on the 
training image with primarily bright leads. Table 3 lists the 25 texture features in the order they were eliminated for each of the three cases: (i) $\mathrm{HH}$ band, (ii) band product and (iii) band ratio.

Accuracy, precision and recall scores in dependence on the number of features eliminated are shown in Figures $4 \mathrm{a}$, b and c, respectively. Dashed lines show accuracy, precision and recall rates for the training set, solid lines are given for the corresponding metrics calculated on the test dataset. Blue lines stand for classification of the band product, green lines stand for classification of the $\mathrm{HH}$ band (i.e. dark lead cases) and red for the band ratio products (i.e. bright lead case).

Accuracies of the three classifications stay almost constant until the first 9 texture features are eliminated. A noticeable decrease in the classification accuracy appears after 16 texture features are eliminated for both the $\mathrm{HH}$ and the band product classifiers and after 17 for the band ratio classifier. This indicates that the first 9 texture feature for each of the tree classifiers (Table 3) can be eliminated without any harm for classification and as little as 3 to 8 texture feature already can provide good classification results.

The precision and the recall rates of the $\mathrm{HH}$ band and the band product classifiers follow corresponding accuracy trends, but show more variation in amplitude. The recall rate of the band ratio classifier shows an increase after 17 texture features are eliminated, but at the same time the precision of the classification drops.

Although all the 25 features could be used for the lead classification, we remove the ones that do not show significant benefit for the classification result. Based on Figs 4a, $b, c$ the number of texture features equal to 9 has been chosen for classification of the $\mathrm{HH}$ band and the band product, i.e. the first 16 texture features of the first and the second columns in Table 3 are removed from the classification. Although we keep only 9 of the texture features, it should, however, be noticed that one could use up to 16 features if even a little improvement of the classification quality is desired. For the band ratio the last 8 texture feature from Table 3 (the third column) are used for classification in further studies, the first 17 are removed from the band ratio classification.

Since the same dataset is used for both the $\mathrm{HH}$ and the band product classifiers, their performance can be compared. The accuracy of the $\mathrm{HH}$ band classification is only $0.2 \%$ higher than the accuracy of the band product classification. On the other hand, the band product classification shows a higher precision, but a lower recall comparing with the $\mathrm{HH}$ band classifier. Since we do not define whether precision or recall is more important for lead classification, we are not able to make a conclusion on which of the two classifiers shows a better classification quality at this point. In order to find which of the two classifiers, based on the band product and the $\mathrm{HH}$ band, shows better classification quality, the influence of a probability threshold on precision and recall and, therefore, on the lead classification is analysed in the next section.

\section{Optimal probability threshold for classifiers}

The Random Forest Classifier can provide probabilistic classification, which can be analysed as is or be used to obtain the corresponding binary classification by setting a threshold. Here we study the influence of a threshold used to produce the binary classification on the classification quality.

Table 3. Elimination order of texture features, i.e. the lower the number $N$ the least important is the respective feature for the classifier

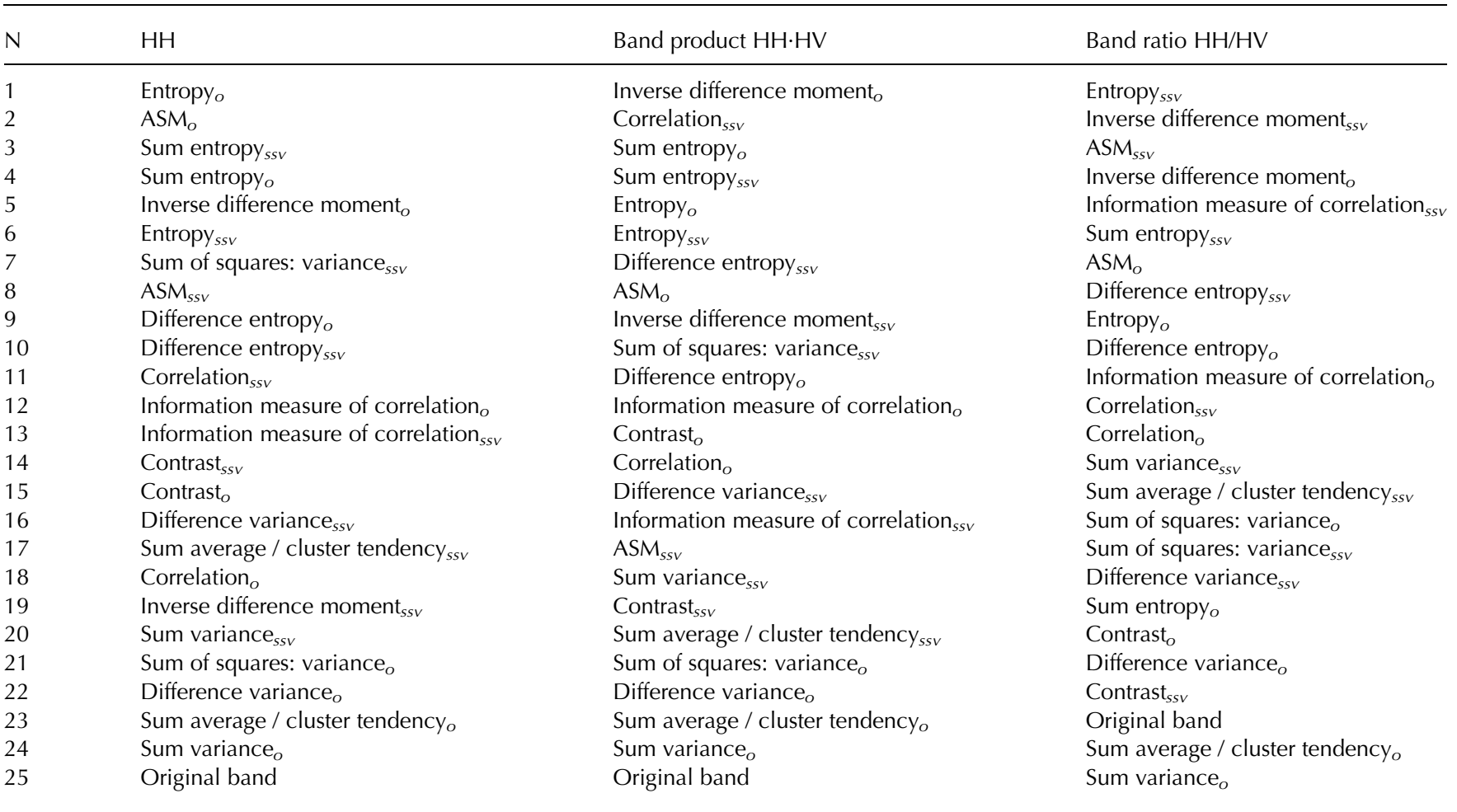

Indices o and ssv stand for the texture features calculated on the original image and the small-scale variations of the image, respectively (see the section 'Texture and polarimetric features'). Definitions of the texture features are given by Haralick and others (1973). The three columns present the elimination order for the three classifiers based on (i) $\mathrm{HH}$, (ii) band product and (iii) band ratio. 


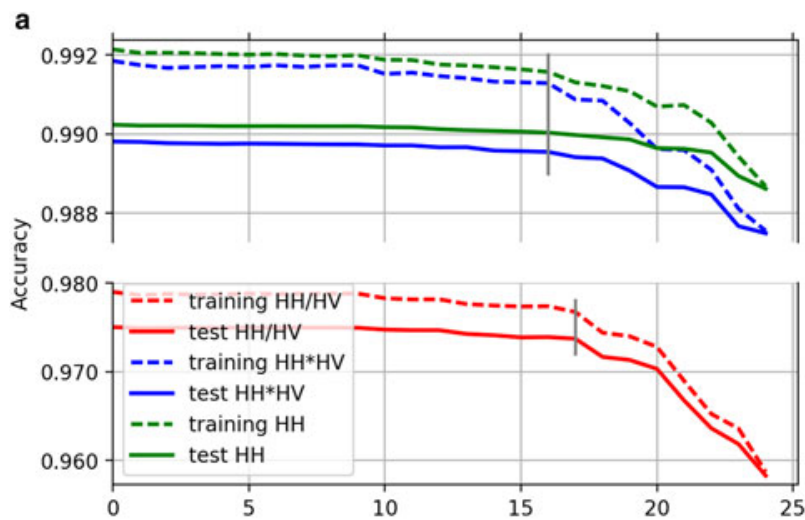

b
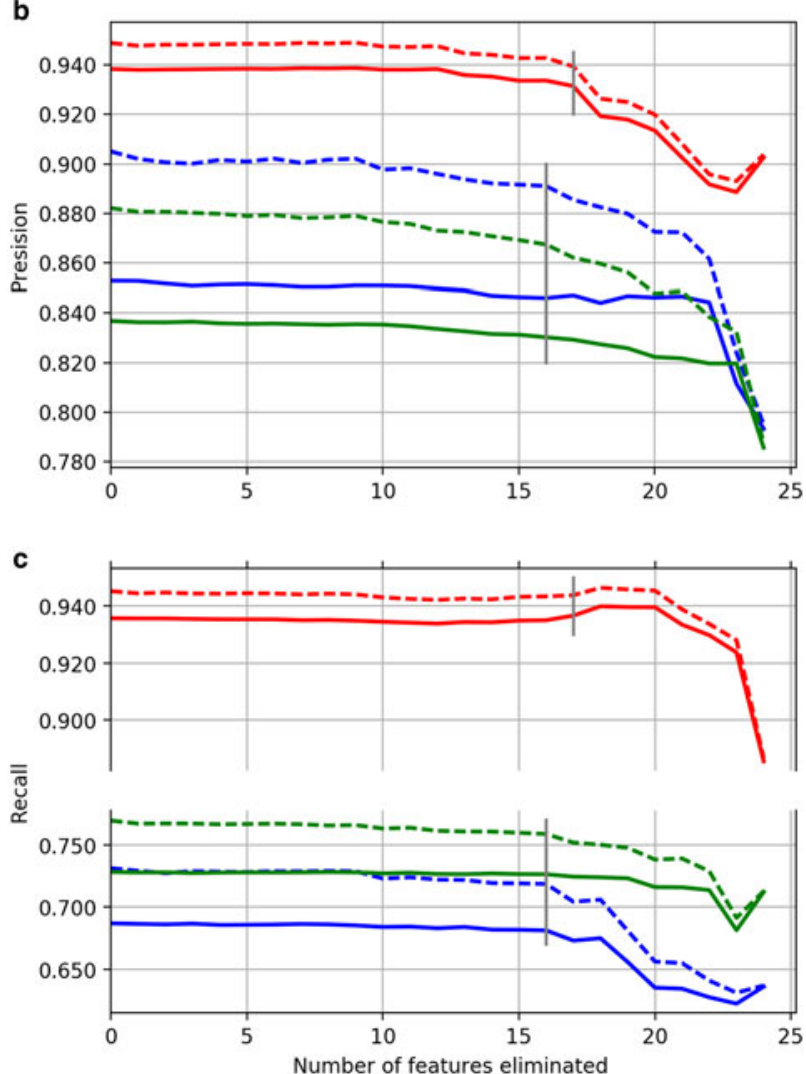

Fig. 4. Accuracy (a), precision (b) and recall (c) scores of the three classifiers depending on number of features eliminated during the RFE analysis. The scores are calculated for the training and test datasets for each of the three classifiers based on the band ratio (red), band product (blue) and $\mathrm{HH}$ band (green).

Three probabilistic classifications of the test data were produced with the three classifiers based on the $\mathrm{HH}$ band, the band product $\mathrm{HH} \cdot \mathrm{HV}$ and the band ratio $\mathrm{HH} / \mathrm{HV}$. Than a range of threshold is applied to each of them to receive the corresponding binary classification.

To describe the influence of the threshold, the precisionrecall curves are calculated for each of the three classifiers (shown in Fig. 5). Dashed lines correspond to precisionrecall curves calculated for the training dataset, solid lines are calculated for the corresponding test dataset. Red lines represent the quality of the classifier based on the band ratio which is used for the bright lead detection. Blue and green lines represent qualities of classifiers based on the band product and the $\mathrm{HH}$ band, respectively. The numbers in black give the respective threshold values used. A higher threshold improves the precision score (i.e. reduce amount

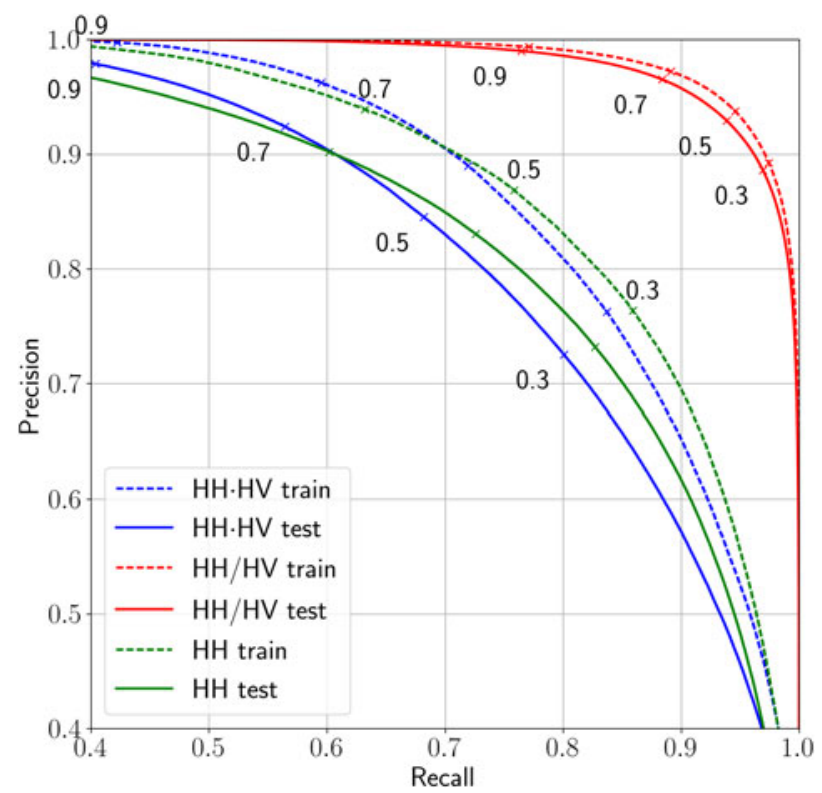

Fig. 5. Precision-recall curves calculated for the training (dashed) and the test (solid) datasets corresponding to three classifiers: based on the band product (blue), the $\mathrm{HH}$ band (green) and the band ratio (red). The curves are obtained by applying different thresholds to a probabilistic classification. The points on the curves which correspond to the threshold values of 30,50, 70 and $90 \%$ are denoted in the figure $(0.3,0.5,0.7$ and 0.9 , respectively).

of misclassification) but at the same time lowers the recall score (reduce the number of lead pixels detected).

From Figure 5 it can be seen that leads are classified with only 84 and $83 \%$ precision on the band product and the $\mathrm{HH}$ band if the default threshold of $50 \%$ probability is used (the solid blue and the solid green curves). But in this case 68 and $72 \%$ of all pixels belonging to the lead class are identified (the recall score). If the threshold is set to $70 \%$, then only 57 and $60 \%$ of the lead pixels will be detected, but precision of the classification is 92 and $90 \%$ (for the band product and the $\mathrm{HH}$ band, respectively). To identify more lead pixels the threshold can be lowered. For example, the threshold set to $30 \%$ will allow to detect 80 and $82 \%$ of all pixels belonging to leads, but the precision of the classification will drop to 72 and $73 \%$, respectively, for the two classifiers $\mathrm{HH}$ and $\mathrm{HH} \cdot \mathrm{HV}$.

Similarly, threshold can be adjusted for the classification of the band ratio. The default value $50 \%$ will give $93 \%$ precision and $94 \%$ recall, the higher value, $70 \%$ for instance, will increase precision to $97 \%$ and decrease recall to $88 \%$. The lower threshold value of $30 \%$ will produce the binary classification with $88 \%$ precision, up to $97 \%$ of lead pixels will be identified.

The solid blue and the solid green curves intersects at the point where precision is $90 \%$ and the recall rate is $60 \%$. This means that the two classifiers show the same classification quality for the corresponding two threshold values of the two classifiers. For a higher threshold value, a higher precision can be achieved with the same recall rate value if the band product classification is used with the appropriate threshold value. Let us consider the right part of precisionrecall curves where the green curve is above the blue. It corresponds to an application where the amount of leads detected (the recall rate) is more important than a very high precision (which is lower than $90 \%$ in this part of the 
curve). In this case, for any value of the recall rate the binary classification based on the $\mathrm{HH}$ band shows better precision (when a certain threshold is applied to the probabilistic classification) than the binary classification based on the band product.

The opposite is true for the left part of precision-recall curves where the blue solid line is above the green one (i.e applications where a high precision is important). Based on these curves one can chose the appropriate threshold value for a certain task. For example, in climate studies about heat balance and energy one would want to have as many leads detected as possible although some of the detections are wrong. On the other hand for navigation, it is more important to know the location of leads with the highest precision possible even though the number of leads detected is lower.

\section{Evaluation}

So far, only results based on the Sentinel-1 SAR data without overlap with optical Sentinel-2 data have been presented. In this section, an evaluation of the leads classification quality is conducted for the two Sentinel-1 SAR scenes which overlap with optical Sentinel-2 data (Figs 2 and 3). Two probabilistic classifications of the first evaluation image (Fig. 2) were produced. The first one is based on the $\mathrm{HH}$ band (Fig. 2a), the second is based on the band product (Fig. 2c); 9 of the most important texture features (last 9 features in the first and the second columns in Table 3 ) were used in both cases. Results are shown in Figure 6 . High probabilities of leads are assigned to areas which can be considered as leads from the optical image. Edges of leads have lower probability comparing to their inner parts. This effect is expected because the classification is based on texture features which are calculated within a window around each pixel. Some ice floes that appear dark on SAR images have non-zero probability values, but can easily be distinguished from leads. The upper part of a lead (the white frame at Figs 6a and b) has a significantly lower probability because it is covered with new ice as it can be confirmed from the optical data (Fig. 2d). Large leads are detected correctly, but thin leads (up to 10 pixels which corresponds to $400 \mathrm{~m}$ ) are often split into several small pieces. Leads with width $<5$ pixels $(200 \mathrm{~m})$ are smoothed on the step of texture features calculation. As the result such leads have low probabilities and can be considered as not-detected. This is a disadvantage of the method which cannot be eliminated because to calculate texture features relevant for a lead, the lead should have a size comparable or larger than the window size used for texture feature computation.

The probabilistic classification of the second evaluation image (Fig. 3c) for the band ratio was performed with 8 input features. Figure 7 shows the result of the probabilistic classification. Many open water areas at the left side of the scene are classified as leads while in the middle leads are not detected. This can be explained by the fact that leads are more pronounced at the left side of the band ratio (Fig. 7c) which was used as input for the algorithm. The fact that areas which are classified as leads are covered with open water can be confirmed from the optical observation (Fig. $7 d$ ).

The following example illustrates the algorithm for lead detection (the flowchart for the algorithm is shown in Fig. 1). The original Sentinel-1 SAR images are shown in Figs $8 \mathrm{a}$ and $\mathrm{b}$. One can observe two large elongated objects, one is dark on both the $\mathrm{HH}$ and the HV bands, the other one is bright on the $\mathrm{HH}$ and dark on the HV band. The two objects represent the two classes of leads we have introduced here, 'bright' and 'dark' leads. No optical data are available for the scene to confirm that the objects are leads, and we can only rely on our experience of how leads appear in SAR images.

To classify both types of leads, two classifiers are applied one for the dark lead detection and one for the bright lead detection. At first, based on the $\mathrm{HH}$ (Fig. 8a) and the HV (Fig. 8b) bands, the band product (Fig. 8c) and the band ratio (Fig. 8d) are calculated. Dark leads are detected from the band ratio using the algorithm based on either the $\mathrm{HH}$ a

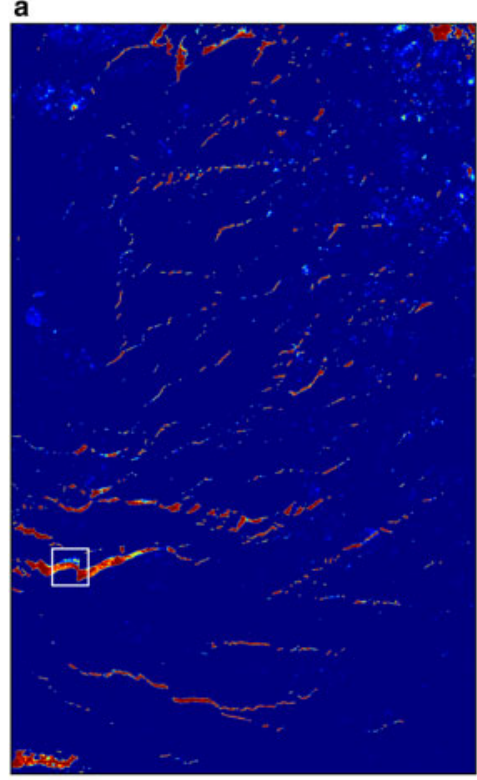

b

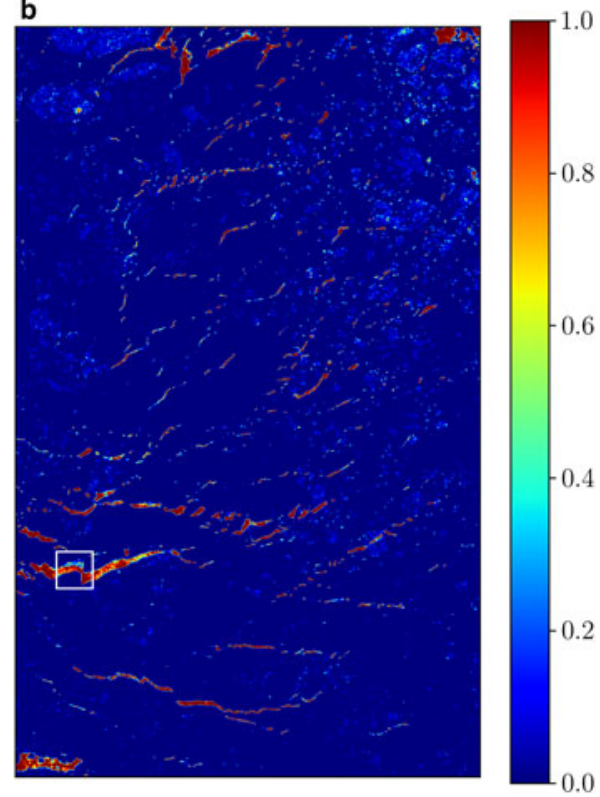

Fig. 6. Probabilistic classifications of the SAR scene shown in Figure 2c performed with the Random Forest Classifier. Panels (a) and (b) are classifications based on the $\mathrm{HH}$ and the band product, respectively. High values mean high probability of a pixel to be classified as a lead. 


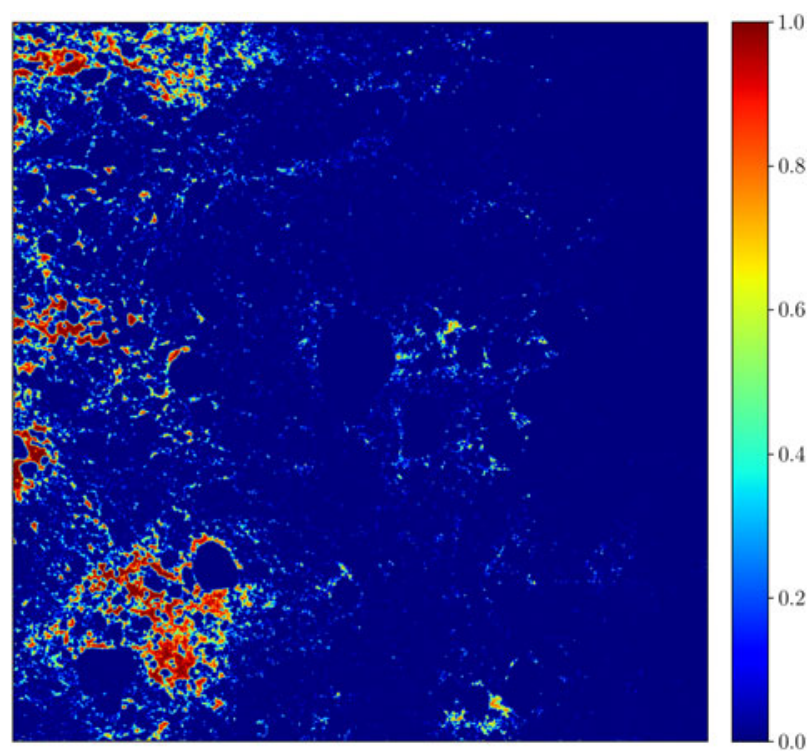

Fig. 7. A probabilistic classification of the scene shown in Figure $3 \mathrm{c}$ performed with the Random Forest Classifier and based on 8 texture features. band or the band product (the result based on the band product is shown in Fig. 8e). Bright leads are detected with the algorithm based on the band ratio (Fig. 8f). Afterwards, the probabilities for the darkand the bright lead branches are added together. As the last step, a threshold of $50 \%$ is applied to the resulting probabilistic classification, so that the final binary classification is obtained (Fig. 8h).

\section{DISCUSSION}

\section{Texture features for classification}

The procedure of texture features elimination shows that the use of a range of texture features (the first 9 from each column of Table 3) do not improve the classification and therefore can be excluded from it. At the same time these texture features do not decrease classification quality. This is because although these features are feed to the classifier, only the texture features that help to perform classification are used within the Random Forest Classifier. In the process of elimination of the next 7-8 texture features, the classification quality slightly decreases. Therefore these 7-8 texture features may also be excluded from the classification process,
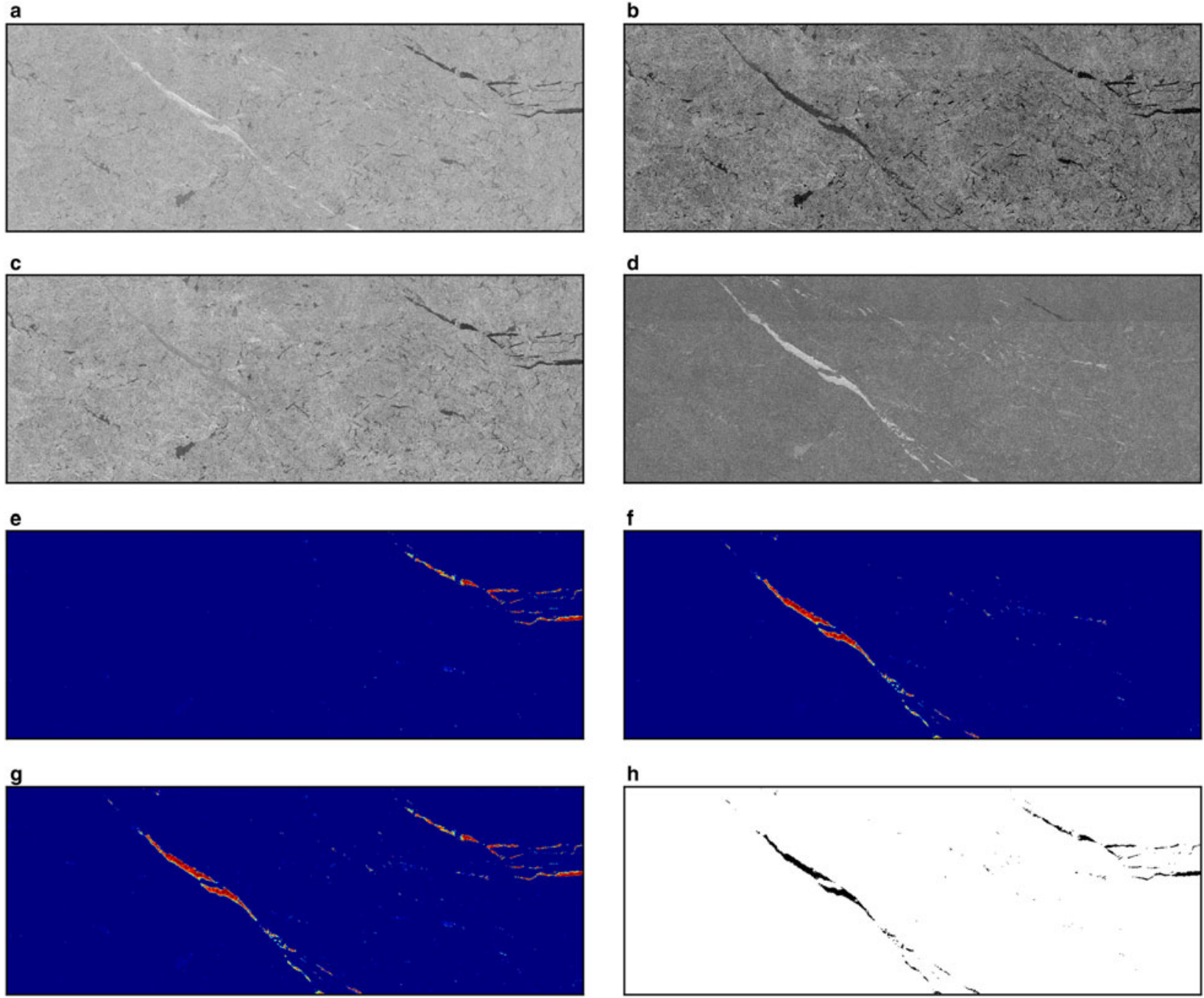

Fig. 8. Panels (a) and (b) are the original HH and HV bands. (c, d) The band product and the band ratio derived from the $\mathrm{HH}$ and $\mathrm{HV}$ bands. (e, f) Probabilistic classifications of the SAR scene based on the band product ratio performed with the Random Forest Classifier. High values means high probability of the pixel to be classified as a lead. (g) The sum of the two probabilistic classification. (h) Binary classification based on (g) with $50 \%$ probability threshold applied. 
especially if fast computation is of importance. As the result 16-17 texture features can be eliminated without significant decrease in classification quality, so that only 8-9 of the texture features are used in classification. In cases when even a little improvement of the classification quality is desired, up to 16 input features can be used. For the case when computational time is limited, the number of texture features used for classification can be decreased.

Of the 9 most important texture features (last 9 rows in Table 3) the majority was derived from the original input band and not from the small-scale variations data, where the background amplitude was removed. The original band is one of the most important inputs for all three classifiers. This means that texture features cannot substitute the original band and provide complementary information. Texture features and the original band should be used in conjunction.

To produce the final lead map a classifier based on either the band product or the $\mathrm{HH}$ band for dark leads should be used in combination with the classifier based on the band ratio for bright leads. In Figure $4 \mathrm{~b}$, the classifier based on the band product shows higher precision; however, in Figure $4 \mathrm{c}$ the recall rate of the classifier is lower compared with the classifier based on the $\mathrm{HH}$ band. This leads us to the conclusion that both the precision and the recall by itself do not provide enough information about the quality of a classification, neither of the two should be used as the only quality metrics, and both of them should be considered together.

In case of probabilistic classification, it is possible to increase the precision by the cost of the recall rate and vice versa by adjusting a threshold used to obtain the corresponding binary classification. Thus, a comparison of the two classifiers is performed by the use of the concept of the precision-recall curve.

\section{Classification quality}

Let us consider the quality of dark leads classification first. The precision-recall curves (Fig. 5) of the $\mathrm{HH}$ and band product classifiers have an intersection point. This means that each of the two classifiers could be considered as the one that should be used in the final product. The quality of the classifier based on the band product is higher when a high precision (over 90\%) of lead detection is desired. In this case, only about $60 \%$ or less leads will be detected. If the recall rate also matters for the application, the $\mathrm{HH}$ band should be used for dark leads classification.

The precision-recall curves clearly show that the quality of bright leads classification is higher than of the dark leads classification based on both the band product and the $\mathrm{HH}$ band (red curves are above blue and green curves). This might be due to the fact that sea ice can appear dark at both the $\mathrm{HH}$ and the HV bands and might be misclassified as a lead. At the same time, the band ratio is known for its good water-sea-ice discrimination potential. Objects, that appear bright at the $\mathrm{HH}$ band and dark at the HV band are usually not misclassified as sea ice (which can be dark or bright at the both bands) or pressure ridges (bright at both bands). This gives us more confidence that an object detected from the band ratio is a lead.

In case of single-band measurements only the $\mathrm{HH}$ band is available for Sentinel-1 images taken in the extra wide swath mode. This single-band mode is often used for scenes at high latitudes. In this case, only the $\mathrm{HH}$ classifier for dark leads can be applied. If dual-band SAR data are available, we are able to calculate the band product and band ratio and therefore to use the classifiers based on $\mathrm{HH} \cdot \mathrm{HV}$ and $\mathrm{HH} / \mathrm{HV}$. The first classifier shows better classification quality for dark leads than the classifier based on the $\mathrm{HH}$ band (Figs. 4 and 5) when used for a high-precision classification (with precision above $90 \%$ ). Since bright leads show a similar backscatter as ridges in $\mathrm{HH}$ band, they cannot be detected from $\mathrm{HH}$ band alone. Thus, with the use of the band ratio more leads are detected from the dual-band SAR data than from single $\mathrm{HH}$ band data.

As the lead detection algorithm is based on backscatter analysis, water between ice floes is classified as leads. During summer the separation of leads from melt ponds will not be possible with the features used here (the shape of the features would have to be taken into account). For the data used in this study there is no evidence for the presence of melt ponds. Therefore the algorithm has not been evaluated for the summer season. The ability of lead classification during the melt season is the subject of future studies.

\section{SUMMARY}

In this study, we developed a lead classification algorithm that can be applied to Sentinel-1 SAR dual band products. To account for the variability of SAR lead signatures in Cband backscatter, two classifiers, one for leads appearing dark (a classifier based on either the $\mathrm{HH}$ band or the band product can be used) and one for leads appearing bright in SAR imagery, were trained. The polarimetric features, the band product $\mathrm{HH} \cdot \mathrm{HV}$ and the band ratio $\mathrm{HH} / \mathrm{HV}$ as well as the $\mathrm{HH}$ band, were extended with 24 texture features each. The texture features are based on the GLCM: 12 of them are calculated for the polarimetric feature directly and another 12 are derived from the small-scale variations of the polarimetric feature. Together with the original polarimetric feature this yields 25 different features that can be feed into the classifier (see Table 3). A RFE procedure showed the texture feature importance for the classification of leads. We found that although all 25 input features can be used, decreasing of their number does not always leads to a decrease of the classification quality. As little as 3 texture features can already provide useful classification of leads. Classifications with 9 texture features for dark and bright leads already show good classification quality and therefore were used further in this study. More features did not significantly improve the classification results.

Precision-recall curves were calculated for the three classifiers based on the $\mathrm{HH}$ band, the band product and the band ratio. For classification of dark leads in the domain of high precision and low recall rates (precision above $90 \%$ and recall below $60 \%$ ) the classification based on the band product shows better quality than the classification based on the $\mathrm{HH}$ band. However, when the amount of leads detected is important, the classifier based on the $\mathrm{HH}$ band shows better quality and therefore is preferred. The classifier based on the band ratio, allows us to identify bright leads that can be detected only if dual band SAR images are available. Therefore, more leads are identified from the combination of the $\mathrm{HH}$ and the $\mathrm{HV}$ bands comparing to the single $\mathrm{HH}$ band SAR product. The classifier is able to identify leads with a high precision of above $95 \%$ for bright leads and above 90\% for dark leads when $70 \%$ of all leads are detected. The overall precision is between the two and depends on ratio of dark and bright leads. The ratio depends on combination of the lead 
distribution, incidence angle, time difference between formation of a lead and acquisition of image over the area with the lead. The precision can be increased by the cost of the amount of leads detected (Fig. 4). The classification of leads from SAR is confirmed by optical imagery from Sentinel-2. Under some circumstance, however, to achieve such high precision the percentage of the total leads area identified (recall score) can drop to $60 \%$ and lower if a higher precision is desired. The advantage of the presented method is that the interplay between precision and recall score can be easily adjusted depending on the application.

The lead detection method presented in this paper has proven to be stable in our tests and the eight SAR scenes presented here (Table 1). In a next step, we will apply this method to a larger amount of Sentinel-1 SAR scenes. The spatial and temporal coverage of Sentinel-1 data in the Arctic will result in lead maps of the European Arctic on a daily basis. With the now operational two Sentinel-1 satellites, $a$ and $b$, the complete Arctic Ocean is covered at least once per week. The Sentinel-1 time series is still short but we expect that lead statistics can already be derived for the first years and extended in future.

\section{ACKNOWLEDGMENTS}

This study was supported by the Institutional Strategy of the University of Bremen, funded by the German Excellence Initiative, and by the Deutsche Forschungsgemeinschaft (DFG) through the International Research Training Group IRTG 1904 ArcTrain. We gratefully acknowledge the provision of Copernicus Sentinel data [2015-2017] provided by the European Union via the Copernicus Open Access Hub (scihub.copernicus.eu).

\section{REFERENCES}

Alonso-Gonzalez A, Lopez-Martnez C, Salembier P and Deng X (2013) Bilateral distance based filtering for polarimetric SAR data. Remote Sens. (Basel), 5, 5620-5641 (doi: 10.3390/rs5115620)

Assmy P and 40 others (2017) Leads in Arctic pack ice enable early phytoplankton blooms below snow-covered sea ice. Sci. Rep., 7 (doi: 10.1038/srep40850)

Breiman L (2001) Random forests. Mach. Learn., 45, 5-32 (doi: 10.1023/A:1010933404324)

Brekke C, Holt B, Jones C and Skrunes S (2014) Discrimination of oil spills from newly formed sea ice by synthetic aperture radar. Remote Sens. Environ., 145, 1-14, (doi: 10.1016/j.rse.2014.01.015)

Brekke C, Jones CE, Skrunes S, Holt B and Espeseth M (2016) Crosscorrelation between polarization channels in SAR imagery over oceanographic features. IEEE Trans. Geosci. Remote Sens., 13 (7), 997-1001 (doi: 10.1109/LGRS.2016.2558543)

Bröhan D and Kaleschke L (2014) A nine-year climatology of arctic sea ice lead orientation and frequency from AMSR-E. Remote Sens. (Basel), 6, 1451-1475, (doi: 10.3390/rs6021451)

Davis J and Goadrich M (2006) The relationship between precisionrecall and ROC curves. In Proc. of the 23 rd Int. Conf. on Machine Learning pp. 233-240

Dierking W (2010) Mapping of different sea ice regimes using images from sentinel-1 and ALOS synthetic aperture radar. IEEE Trans. Geosci. Remote Sens., 48(3), 1045-1058, (doi: 10.1109/TGRS.2009.2031806)

Dierking W (2013) Sea ice monitoring by synthetic aperture Radar. Oceanography, 26(3), 100-111 (doi: 10.5670/oceanog.2013.33)

Dierking W and Wesche C (2014) C-Band Radar polarimetry - useful for detection of icebergs in sea ice?. IEEE Trans. Geosci. Remote Sens., 52(1), 25-37 (doi: 10.1109/TGRS.2012.2234756)
ESA (2016) Sentinel-1 Level 1 Detailed Algorithm Definition. Technical Report, ESA

Fawcett T (2006) An introduction to ROC analysis. Pattern Recognit. Lett., 27, 861-874. (doi: 10.1016/j.patrec.2005.10.010)

Haralick RM, Shanmugam K and Dinstein I (1973) Textural Features for Image Classification. IEEE Trans. Syst. Man Cybern., smc-3(6), 610-621

Isleifson D, 5 others (2014) A study on the C-Band polarimetric scattering and physical characteristics of frost flowers on experimental sea ice. IEEE Trans. Geosci. Remote Sens., 52(3), 1787-1798 (doi: 10.1109/TGRS.2013.2255060)

Ivanova N, Rampal P and Bouillon S (2016) Error assessment of satellite-derived lead fraction in the Arctic. Cryosphere, 10, 585-595, ISSN 19940424 (doi: 10.5194/tc-10-585-2016)

Karvonen J (2014) Baltic sea ice concentration estimation based on C-band dual-polarized SAR data. IEEE Trans. Geosci. Remote Sens., 52(9), 5558-5566, (doi: 10.1109/TGRS.2013.2290331)

Korosov AA and Park JW (2016) Very high resolution classification of Sentinel-1A data using segmentation and texture analysis. In Living Planet Symp. 2016 p. 44

Leigh S, Wang Z and Clausi DA (2014) Automated ice-water classification using dual polarization SAR satellite imagery. IEEE Trans. Geosci. Remote Sens., 52(9), 5529-5539, (doi: 10.1109/TGRS.2013.2290231)

Lindsay RW and Rothrock DA (1995) Arctic sea ice leads from advanced very high resolution radiometer images. J. Geophys. Res., 100(C3), 4533-4544 (doi: 10.1029/94JC02393)

Liu H, Guo H and Zhang L (2015) SVM-Based sea ice classification using textural features and concentration from RADARSAT-2 Dual-Pol ScanSAR data. IEEE J. Sel. Top. Appl. Earth Observ. Remote Sens., (doi: 10.1109/JSTARS.2014.2365215)

Maykut GA (1978) Energy exchange over young sea ice in the central Arctic. J. Geophys. Res., 83(C7), 3646-3658. (doi: 10. 1029/JC083iC07p03646)

Moen MAN, Anfinsen SN, Doulgeris AP, Renner $\mathrm{AHH}$ and Gerland S (2015) Assessing polarimetric SAR sea-ice classifications using consecutive day images. Ann. Glaciol., 56(69), 285-294, (doi: 10.3189/2015AoG69A802)

Nghiem SV, 5 others (1997) A laboratory study of the effect of frost flowers on $\mathrm{C}$ band radar backscatter from sea ice. J. Geophys. Res., 102(C2), 3357-3370 (doi: 10.1029/96JC03208)

Pedregosa F, 15 others (2011) Scikit-learn: Machine Learning in Python. J. Mach. Learn. Res., 12, 2825-2830.

Ressel R, Singha S, Lehner S, Rosel A and Spreen G (2016) Investigation into different polarimetric features for sea ice classification using X-band synthetic aperture radar. IEEE J. Sel. Top. Appl. Earth Observ. Remote Sens., 9(7), 3131-3143, (doi: 10.1109/JSTARS.2016.2539501)

Röhrs J and Kaleschke L (2012) An algorithm to detect sea ice leads by using AMSR-E passive microwave imagery. Cryosphere, 6(0), 343-352, (doi: 10.5194/tc-6-343-2012)

Scharien RK and Yackel JJ (2005) Analysis of surface roughness and morphology of first-year sea ice melt ponds: implications for microwave scattering. IEEE Trans. Geosci. Remote Sens., 43 (12), 2927-2939. (doi: 10.1109/TGRS.2005.857896)

Spreen G, Kaleschke L and Heygster G (2008) Sea ice remote sensing using AMSR-E 89-GHz channels. J. Geophys. Res., 113, (doi: 10.1029/2005JC003384)

Steffen K (1991) Energy flux density estintation over sea ice based on satellite passive microwave measurements. Ann. Glaciol., 15, 178-183.

Tomasi C and Manduchi R (1998) Bilateral filtering for gray and color images. In Sixth International Conference on Computer Vision, pp. 839-846 (doi: 10.1109/ICCV.1998.710815)

Wang Q, Danilov S, Jung T, Kaleschke L and Wernecke A (2016) Sea ice leads in the Arctic Ocean: Model assessment, interannual variability and trends. Geophys. Res. Lett., 43, 7019-7027, (doi: 10.1002/2016GL068696)

Weeks W (2010) On Sea Ice. University of Alaska Press, Fairbanks, Wernecke A and Kaleschke L (2015) Lead detection in Arctic sea ice from CryoSat-2: Quality assessment, lead area fraction and 
width distribution. Cryosphere, 9, 1955-1968, (doi: 10.5194/tc9-1955-2015)

Willmes S and Heinemann G (2015) Pan-Arctic lead detection from MODIS thermal infrared imagery. Ann. Glaciol., 56(69), 29-37 (doi: 10.3189/2015AoG69A615)

Willmes S and Heinemann G (2016) Sea-ice wintertime lead frequencies and regional characteristics in the Arctic, 2003-2015. Remote Sens. (Basel), 8(4), (doi: 10.3390/rs8010004)
Zakhvatkina N, Korosov A, Muckenhuber S, Sandven S and Babiker M (2017) Operational algorithm for icewater classification on dual-polarized RADARSAT-2 images. Cryosphere, 11, 33-46 (doi: 10.5194/tc-11-33-2017)

Zakhvatkina NY, Alexandrov VY, Johannessen OM, Sandven S and Frolov IY (2013) Classification of sea ice types in ENVISAT synthetic aperture radar images. IEEE Trans. Geosci. Remote Sens., 51(5), 2587-2600 (doi: 10.1109/TGRS.2012.2212445) 